\title{
Medicinal plants used in Northern Peru for reproductive problems and female health
}

\author{
Rainer W Bussmann*, Ashley Glenn
}

\begin{abstract}
Infections of the reproductive tract, complications after childbirth, and reproductive problems continue to be a major health challenge worldwide. An impressive number of plant species is traditionally used to remedy such afflictions, and some have been investigated for their efficacy with positive results. A total of 105 plant species belonging to 91 genera and 62 families were documented and identified as herbal remedies for reproductive problems in Northern Peru. Most species used were Asteraceae (9.52\%), followed by Lamiaceae and Fabaceae (8.57\% and 6.67\%). The most important families are clearly represented very similarly to their overall importance in the local pharmacopoeia. The majority of herbal preparations for reproductive afflictions were prepared from the leaves of plants $(22.72 \%)$, the whole plant $(21.97 \%)$, and stems $(21.21 \%)$, while other plant parts were used less frequently. More than $60 \%$ of the cases fresh plant material was used to prepare remedies. Over $70 \%$ of the remedies were applied orally, while the remaining ones were applied topically. Many remedies were prepared as mixtures of multiple ingredients.

Little scientific evidence exists to prove the efficacy of the species employed as reproductive disorder remedies in Northern Peru. Only 34\% of the plants found or their congeners have been studied at all for their medicinal properties. The information gained on frequently used traditional remedies might give some leads for future targets for further analysis in order to develop new drugs.
\end{abstract}

\section{Background}

According to 1999 WHO estimates reproductive problems, including, 340 million new cases of curable Sexually Transmitted Diseases (STIs; syphilis, gonorrhoea, chlamydia and trichomoniasis) occur annually throughout the world in adults aged 15-49 years. In developing countries, STIs and their complications rank in the top five disease categories for which adults seek health care. Infection with STIs can lead to acute symptoms, chronic infection and serious delayed consequences such as infertility, ectopic pregnancy, cervical cancer and the untimely death of infants and adults [1].

Traditional Medicine (TM) is used globally and is rapidly growing in economic importance. In developing countries, TM is often the only accessible and affordable treatment available. The WHO reports that TM is the primary health care system for $80 \%$ of the population in developing countries. In Latin America, the WHO Regional Office for the Americas (AMRO/PAHO) reports that

\footnotetext{
* Correspondence: rainer.bussmann@mobot.org

William L. Brown Center, Missouri Botanical Garden, P.O. Box 299, St. Louis, MO 63166-0299, USA
}

$71 \%$ of the population in Chile and $40 \%$ of the population in Colombia have used TM. The WHO indicates that in many Asian countries TM is widely used, even though Western medicine is often readily available, and in Japan, $60-70 \%$ of allopathic doctors prescribe TMs for their patients [2].

Complementary Alternative Medicine (CAM) is gaining popularity in many developed countries. Forty-two percent of the population in the US have used CAM at least once [3], and the use of at least one of 16 alternative therapies increased from 34\% in 1990 to $42 \%$ in 1997 [4]. The number of visits to providers of CAM now exceeds by far the number of visits to all primary care physicians in the US [5,6]. The expenses for the use of TM and CAM are exponentially growing in many parts of the world. The 1997 out-of-pocket CAM expenditure was estimated at US\$2,7 billion in the USA, and the world market for herbal medicines based on traditional knowledge is now estimated at US\$ 60 billion [7].

Northern Peru is believed to be the center of the Central Andean Health Axis [8], and traditional medicinal practices in this region remain an important component

\section{Biomed Central}


of everyday life [9-13]. TM is also gaining acceptance by national governments and health providers. Peru's National Program in Complementary Medicine and the Pan American Health Organization recently compared Complementary Medicine to allopathic medicine in clinics and hospitals operating within the Peruvian Social Security System. The results showed that the cost of using Traditional Medicine was less than the cost of Western therapy. In addition, for each of the criteria evaluated - clinical efficacy, user satisfaction, and future risk reduction - Traditional Medicine 's efficacy was higher than that of conventional treatments, including fewer side effects, higher perception of efficacy by both the patients and the clinics, and a 53-63\% higher cost efficiency of Traditional Medicine over that of conventional treatments for the selected conditions [14]. According to [6], the sustainable cultivation and harvesting of medicinal species is one of the most important challenges for the next few years.

The present study attempts to give an overview on medicinal plant species employed in Northern Peru in traditional remedies for reproductive problems and female health, and compare this use to the western scientific evidence regarding their efficacy.

\section{Materials and Methods}

\section{Plant Collections}

Plants for the present study were collected in the field, in markets, and at the homes of traditional healers (curanderos) in Northern Peru in 10 2-3 months long field visits between 2001 and 2009, as a larger scale project following initial collections in southern Ecuador (Figure 1). The same 116 informants (healers and market vendors) in the Trujillo and Chiclayo area were repeatedly interviewed during this time, using structured questionnaires. The informants were always provided with fresh (non-dried) plant material, either collected with them, by them, or available at their market stands. The questionnaires did not include any reference as to disease concepts, plant parts or preparations. In contrast, the participants were asked simple questions along the lines "What is this plant used for, which part, which quantity, how is it prepared, are any other plants added to the mixture." All questions were asked in the same order. All informants were of Mestizo origin, and spoke only Spanish as their native language, and all interviews were conducted in Spanish. The study covered the four existing medicinal plant markets of the region, and included all vendors present. All interviews were conducted with the same set of participants. The specimens are registered under the collection series "RBU/PL," "ISA," “GER," “JULS," “EHCHL," "VFCHL," "TRUBH," and "TRUVANERICA," depending on the year of fieldwork and collection location. Surveys were conducted in Spanish by fluent speakers. Surveyors would approach healers, collectors and market vendors and explain the premise for the study, including the goal of conservation of medicinal plants in the area.

Vouchers of all specimens were deposited at the Herbario Truxillensis (HUT, Universidad Nacional de Trujillo), and Herbario Antenor Orrego (HAO, Universidad Privada Antenor Orrego Trujillo). In order to recognize Peru's rights under the Convention on Biological Diversity, most notably with regard to the conservation of genetic resources in the framework of a study treating medicinal plants, the identification of the plant material was conducted entirely in Peru. No plant material collected either in this study in Northern Peru, or the previous study in Southern Ecuador was exported in any form whatsoever.

\section{Species identification and nomenclature}

The nomenclature of plant families, genera, and species follows the Catalogue of the Flowering Plants and Gymnosperms of Peru [15] and the Catalogue of Vascular Plants of Ecuador [16]. The nomenclature was compared to the TROPICOS database. Species were identified using the available volumes of the Flora of Peru [17], as well as [18-20], and the available volumes of the Flora of Ecuador [21].

\section{Results}

A total of 105 plant species belonging to 91 genera and 62 families were documented and identified as herbal remedies for reproductive problems in Northern Peru. Most species used were Asteraceae (9.52\%), followed by Lamiaceae and Fabaceae (8.57\% and 6.67\%). Other families were less important, and 44 contributed only one species each to the pharmacopoeia (Table 1). The most important families are clearly represented very similarly to their overall importance in the local pharmacopoeia (Table 1) [9].

The majority of herbal preparations for reproductive issues were prepared from the leaves of plants $(22.72 \%)$, the whole plant $(21.97 \%)$, and stems $(21.21 \%)$, while other plant parts were used much less frequently (Table 2 ). This indicates that the local healers count on a very well developed knowledge about the properties of different plant parts. In almost $62 \%$ of the cases fresh plant material was used to prepare remedies, which differs little from the average herbal preparation mode in Northern Peru. Over $70 \%$ of the remedies were applied orally, while the remaining ones were applied topically. Many remedies were prepared as mixtures of multiple ingredients by boiling plant material either in water or in sugarcane spirit.

A complete overview of all plants encountered is given in Table 3 . 


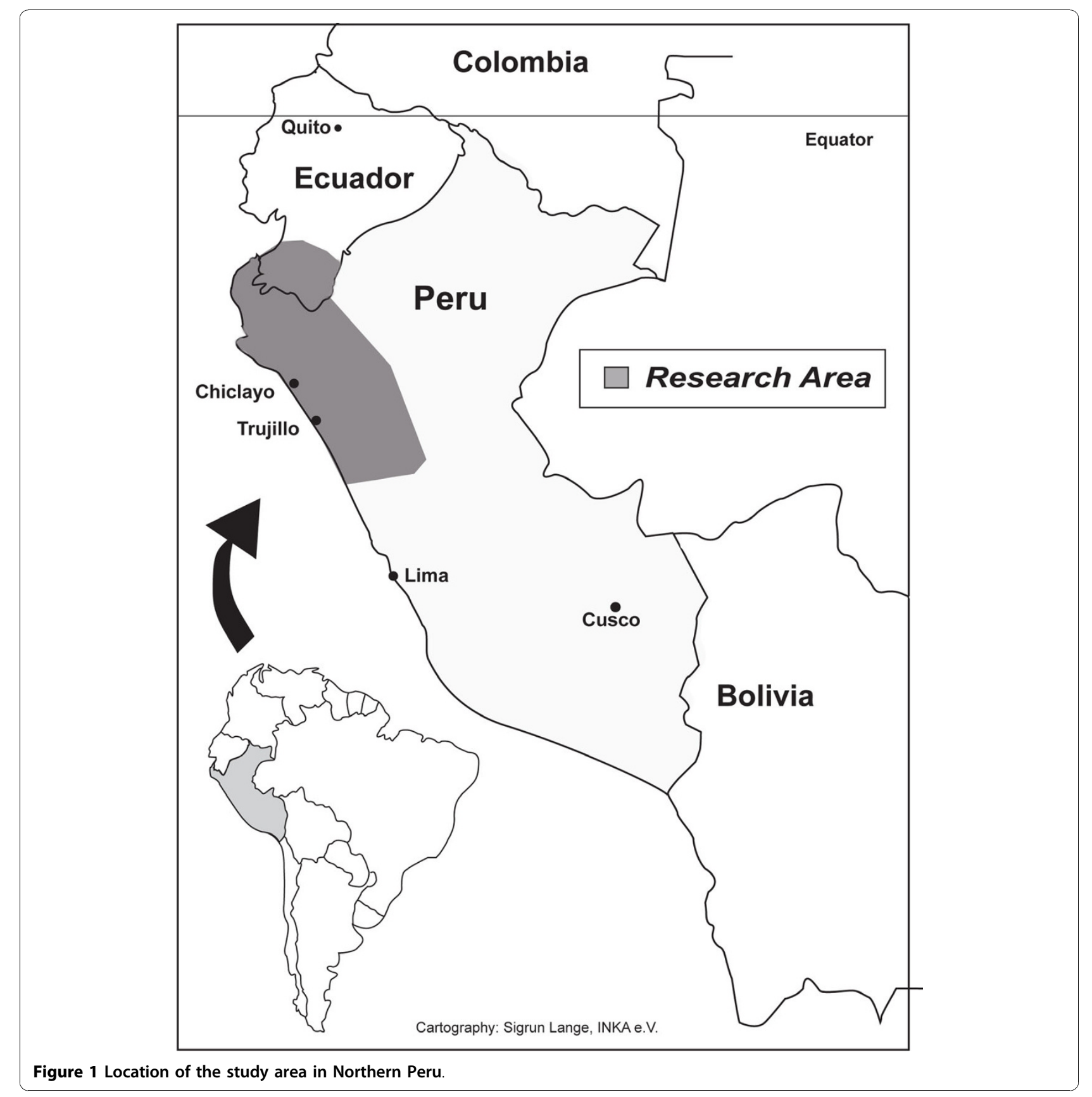

\section{Discussion}

Little scientific evidence exists to prove the efficacy of the species employed as reproductive disorder remedies in Northern Peru. Only 34\% of the plants found or their congeners have been studied at all for their medicinal properties. Aloe spp. are known to have oestrogenic activity [22,23]. [24] reported that Artemisia spp. had effects on female health amongst the Cumash. A variety of other Asteraceae has been shown to be used against menopausal symptoms (Clibadium: [25]; Matricaria: [26-28]; Taraxacum: [29,30]. [23] found hormonal effects in Cordia sp., while [31-35] reported on anti-fertility effects of Dioscorea sp. Cupressus sp. are well known abortifacients (e.g. [36]), while pumpkin seed oil showed testosterone-inhibitory effects (e.g. [23,37-39]). Chamaesyce sp. showed promise in the treatment of male infertility, while Mimosa sp. on the contrary are used to reduce spermal fertility $[23,40]$.

A wide range of Lamiaceae have been shown to exhibit contraceptive efficacy, and the same species are used in Peru for similar purposes (Mentha spp.: [41-44]; Ocimum spp.: [45-48]; Origanum majorana: [44,49,50]; Rosmarinus 
Table 1 Plants used for reproductive issues in Northern Peru and Comparison of reproductive treatments to the ten most important plant families of the medicinal flora of Northern Peru (after Bussmann \& Sharon 2006)

Family Genera Species $\% \begin{gathered}\text { Medicinal flora of } \\ \text { Northern Peru (most } \\ \text { important families) }\end{gathered}$

\begin{tabular}{|c|c|c|c|c|}
\hline Asteraceae & 9 & 10 & 9.52 & 13.64 \\
\hline Lamiaceae & 7 & 9 & 8.57 & 4.87 \\
\hline Fabaceae & 6 & 7 & 6.67 & 6.82 \\
\hline Solanaceae & 2 & 4 & 3.81 & 4.09 \\
\hline Poaceae & 3 & 3 & 2.84 & 2.33 \\
\hline Cucurbitaceae & 1 & 3 & 2.84 & 1.75 \\
\hline Plantaginaceae & 1 & 3 & 2.84 & \\
\hline Amaranthaceae & 2 & 2 & 1.92 & \\
\hline Anacardiaceae & 2 & 2 & 1.92 & \\
\hline Boraginaceae & 2 & 2 & 1.92 & \\
\hline Brassicaceae & 2 & 2 & 1.92 & \\
\hline Euphorbiaceae & 2 & 2 & 1.92 & 2.33 \\
\hline Olacaceae & 2 & 2 & 1.92 & \\
\hline Rutaceae & 2 & 2 & 1.92 & \\
\hline Dioscoreaceae & 1 & 2 & 1.92 & \\
\hline Geraniaceae & 1 & 2 & 1.92 & \\
\hline Linaceae & 1 & 2 & 1.92 & \\
\hline Passifloraceae & 1 & 2 & 1.92 & \\
\hline Adiantaceae & 1 & 1 & 0.95 & \\
\hline Alstroemeriaceae & 1 & 1 & 0.95 & \\
\hline Amaryllidaceae & 1 & 1 & 0.95 & \\
\hline Apiaceae & 1 & 1 & 0.95 & 2.14 \\
\hline Apocynaceae & 1 & 1 & 0.95 & \\
\hline Asclepiadaceae & 1 & 1 & 0.95 & \\
\hline Asphodelaceae & 1 & 1 & 0.95 & \\
\hline Balanophoraceae & 1 & 1 & 0.95 & \\
\hline Bignoniaceae & 1 & 1 & 0.95 & \\
\hline Cactaceae & 1 & 1 & 0.95 & \\
\hline Convolvulaceae & 1 & 1 & 0.95 & \\
\hline Cupressaceae & 1 & 1 & 0.95 & \\
\hline Cyperaceae & 1 & 1 & 0.95 & \\
\hline Dipsacaceae & 1 & 1 & 0.95 & \\
\hline Ericaceae & 1 & 1 & 0.95 & \\
\hline Erythroxylaceae & 1 & 1 & 0.95 & \\
\hline Gentianaceae & 1 & 1 & 0.95 & \\
\hline Illiciaceae & 1 & 1 & 0.95 & \\
\hline Isoetaceae & 1 & 1 & 0.95 & \\
\hline Krameriaceae & 1 & 1 & 0.95 & \\
\hline Lauraceae & 1 & 1 & 0.95 & \\
\hline Loganiaceae & 1 & 1 & 0.95 & \\
\hline Loranthaceae & 1 & 1 & 0.95 & \\
\hline Lythraceae & 1 & 1 & 0.95 & \\
\hline Malvaceae & 1 & 1 & 0.95 & \\
\hline Menispermaceae & 1 & 1 & 0.95 & \\
\hline Moraceae & 1 & 1 & 0.95 & \\
\hline Myristicaceae & 1 & 1 & 0.95 & \\
\hline
\end{tabular}

Table 1 Plants used for reproductive issues in Northern Peru and Comparison of reproductive treatments to the ten most important plant families of the medicinal flora of Northern Peru (after Bussmann \& Sharon 2006) (Continued)

\begin{tabular}{|c|c|c|c|c|}
\hline Nyctaginaceae & 1 & 1 & 0.95 & \\
\hline Orchidaceae & 1 & 1 & 0.95 & \\
\hline Oxalidaceae & 1 & 1 & 0.95 & \\
\hline Polygonaceae & 1 & 1 & 0.95 & \\
\hline Polypodiaceae & 1 & 1 & 0.95 & \\
\hline Portulacaceae & 1 & 1 & 0.95 & \\
\hline Proteaceae & 1 & 1 & 0.95 & \\
\hline Ranunculaceae & 1 & 1 & 0.95 & \\
\hline Rosaceae & 1 & 1 & 0.95 & 1.75 \\
\hline Rubiaceae & 1 & 1 & 0.95 & \\
\hline Thelypteridaceae & 1 & 1 & 0.95 & \\
\hline Thymeleaceae & 1 & 1 & 0.95 & \\
\hline Typhaceae & 1 & 1 & 0.95 & \\
\hline Urticaceae & 1 & 1 & 0.95 & \\
\hline Valerianaceae & 1 & 1 & 0.95 & \\
\hline Verbenaceae & 1 & 1 & 0.95 & \\
\hline Lycopodiaceae & 0 & 0 & 0.00 & 1.95 \\
\hline TOTAL & 91 & 105 & 100 & \\
\hline
\end{tabular}

officinalis: [40]). Similar efficacy has been shown for Sanguisorba officinalis [51], and Ruta graveolens [23,52-55].

Various species of Passiflora have aphrodisiac activity [56-60], and Myristica fragrans as well as Syzygium aromaticum [61,62], and extracts of Lantana camara $[63,64]$ and Pilea spp. [23] fulfil the same purpose, while Portulaca oleracea showed efficacy in relieving uterine bleeding $[65,66]$.

\section{Conclusions}

Infections of the reproductive tract, complications after childbirth, and reproductive problems continue to be a major health challenge worldwide. An impressive number of plant species is traditionally used to remedy such

Table 2 Plant part used

\begin{tabular}{lrr}
\hline Plant part & $\%$ & Species \\
\hline Leaves & 22.72 & 30 \\
Whole plant & 21.97 & 29 \\
Stems & 21.21 & 28 \\
Flowers & 9.85 & 13 \\
Root & 8.33 & 11 \\
Seeds & 6.82 & 9 \\
Bark & 4.55 & 6 \\
Fruit & 2.27 & 3 \\
Latex & 1.52 & 2 \\
Wood & 0.76 & 1 \\
\hline
\end{tabular}


Table 3 Species encountered and used in Northern Peru for reproductive problems

\begin{tabular}{|c|c|c|c|c|c|}
\hline $\begin{array}{l}\text { Family/Genus/ } \\
\text { Species }\end{array}$ & Indigenous name & $\begin{array}{l}\text { Plant part } \\
\text { used }\end{array}$ & Admin. & Use & Coll. \# \\
\hline \multicolumn{6}{|l|}{ ADIANTACEAE } \\
\hline $\begin{array}{l}\text { Adiantum concinnum } \\
\text { Wild. ex H.B.K. }\end{array}$ & $\begin{array}{l}\text { Culantrillo del Pozo, } \\
\text { Culantrillo }\end{array}$ & $\begin{array}{l}\text { Leaves and } \\
\text { Stems, fresh } \\
\text { or dried }\end{array}$ & Oral & Menstrual regulation & $\begin{array}{l}\text { VFCHL29, TRUBH17, RBU/ } \\
\text { PL265, JULS149 }\end{array}$ \\
\hline
\end{tabular}

\section{AMARANTHACEAE}

Alternanthera

Sanguinaria, Moradilla, porrigens (Jacquin) Lancetilla

Kuntze

Iresine diffusa H.B.K. ex Paja Blanca, Sangrinaria Willd.

\section{ALSTROEMERIACEAE}

Bomarea angustifolia Benth.

Cachuljillo

\section{AMARYLLIDACEAE}

Eustephia coccinea

Cav.

\section{ANACARDIACEAE}

Mauria heterophylla H. Shimir, Tres Hojas, Trinidad, B.K. Chacur, Ahimir, Feregreco

Schinus molle L.

Molle, Moy

\section{APIACEAE}

Petroselinum crispum Perejil

(Miller) A.W. Hill

\section{APOCYNACEAE}

Thevetia peruviana (Pers.) Schum

Mailchin, Maichil, Camalonga, Cabalonga

\section{ASCLEPIADACEAE}

Sarcostemma clausum Marrajudio (Jacquin) Schultes

\section{ASPHODELACEAE}

Aloe vera (L.) Burm $\mathrm{f}$.

\section{ASTERACEAE}

Ambrosia peruviana Willd.

Artemisia absinthium

Chuquiraga spinosa sp. huamanpinta C. Ezcurra

Clibadium cf. sylvestre Flor de Novia

(Aubl.) Baill.

$\begin{array}{ll}\begin{array}{l}\text { Matricaria frigidum } \\ \text { (HBK) Kunth }\end{array} & \text { Manzanilla } \\ \text { Matricaria recutita L. } & \text { Manzanillon, Agua de la } \\ & \begin{array}{l}\text { Banda, Manzanilla Blanca, } \\ \text { Manzanilla Amarga, } \\ \\ \text { Manzanilla }\end{array}\end{array}$
Sabila, Aloe Vera

Altamisa, Marco, Artamisa, Manzanilla del Muerto, Ajenjo, Llatama Negra Malera, Llatama Roja Malera

Ajenco
Whole plant, Topical Cleansing womb after childbirth EHCHL142, ISA56, RBU/ fresh or dried

PL301, RBU/PL324,

EHCHL93, GER117

Whole plant, Oral Inflammation of the ovaries, Menstruation JULS75, ISA62

fresh symptoms in adolescents

Whole plant, Oral Infertility in women

ISA27 dried

Bark, fresh or Oral Inflammation of uterus dried

RBU/PL313, GER71, EHCHL68

Leaves, fresh 1. Oral 1. Inflammation of uterus, Inflammation 2. of the ovaries, Cysts, Fibroids Topical 2. Vaginal cleansing

Bark and Topical Vaginal infection

Latex, fresh

Whole plant, Oral Regulation of menstrual cycle fresh RBU/PL278, JULS225

Seeds, dried Oral Menopause

HCHL162, TRUVan/ Erica19, JULS187, EHCHL174, GER225

Leaves, Oral Promoting lactation in women after birth JULS121, GER43

Stems, fresh

Sabila, Zabila, Aloe, Hojas de

Leaves, fresh

Topcial Vaginal inflammation, Vaginal ulcers, Vaginal cance

UUL274, GER22

Leaves and

Stems, fresh

Topical After birth to reduce inflamation and prevent spasms in the woman's womb

Whole plant, Oral Menstrual colics, Menstration, Regulating preferably Leaves and the menstrual cycle

EHCHL165, VFCHL10

JULS108, TRUBH18, RBU/ PL370, TRUBH15, JULS90, GER9, GER110

ISA66, RBU/PL363, GER146

Stems, fresh Leaves, dried

Oral

Prostate, Prostate inflammation, Sexual

EHCHL168, TRUBH9, impotence JULS276, RBU/PL373

Flowers, Topical Before marriage

EHCHL80

Leaves and

Stems, fresh or dried

Whole plant, Topical Inflammation of the vagina

JULS22, EHCHL1, TRUBH7 fresh or dried

Whole plant, Topical 1. Vaginal cleansing

fresh 2. Menstrual colics

JULS192, RBU/PL306, ISA120, ISA76, GER145 
Table 3 Species encountered and used in Northern Peru for reproductive problems (Continued)

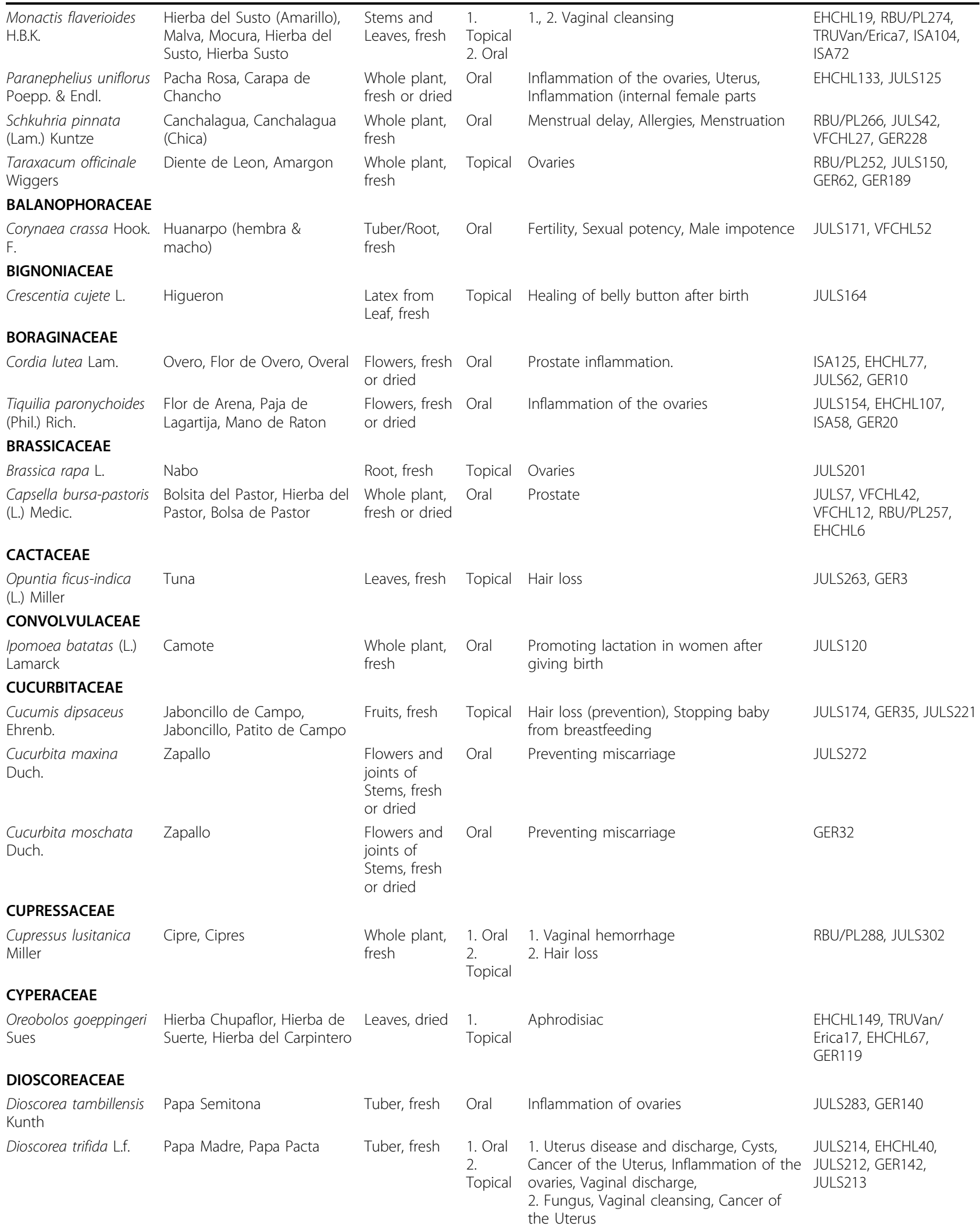


Table 3 Species encountered and used in Northern Peru for reproductive problems (Continued)

\begin{tabular}{|c|c|c|c|c|c|}
\hline \multicolumn{6}{|l|}{ DIPSACACEAE } \\
\hline $\begin{array}{l}\text { Scabiosa atropurpurea } \\
\text { L. }\end{array}$ & $\begin{array}{l}\text { Ambarina, Ambarina Negra, } \\
\text { Flor de Ambarina }\end{array}$ & Flowers, fresh & $\begin{array}{l}\text { 1. Oral } \\
2 . \\
\text { Inhaled }\end{array}$ & Menstrual regulation & $\begin{array}{l}\text { JULS100, EHCHL111, } \\
\text { RBU/PL372, ISA50 }\end{array}$ \\
\hline \multicolumn{6}{|l|}{ ERICACEAE } \\
\hline Bejaria aestuans L. & $\begin{array}{l}\text { Pullunrosa, Cadillo, Payama, } \\
\text { Hierba de la Postema, } \\
\text { Purenrosa, Rosada, Hierba } \\
\text { del buen querer }\end{array}$ & $\begin{array}{l}\text { Flowers, } \\
\text { Leaves and } \\
\text { Stems, fresh } \\
\text { or dried }\end{array}$ & 1. Oral & $\begin{array}{l}\text { Prostate, Menstrual regulation, } \\
\text { Inflammation of uterus, Cysts, } \\
\text { Inflammation of ovaries, Inflammation of } \\
\text { the womb, Uterus, Menstrual pain }\end{array}$ & $\begin{array}{l}\text { VFCHL22, JULS50, } \\
\text { EHCHL39, ISA114, ISA43, } \\
\text { JULS234, GER121 }\end{array}$ \\
\hline \multicolumn{6}{|l|}{ ERYTHROXYLACEAE } \\
\hline Erythroxylon coca Lam. & Coca & Leaves, dried & Oral & $\begin{array}{l}\text { Induce child birth, Strength for woman } \\
\text { during childbirth, Helping delivery of } \\
\text { newborn }\end{array}$ & JULS144, GER201 \\
\hline \multicolumn{6}{|l|}{ EUPHORBIACEAE } \\
\hline $\begin{array}{l}\text { Chamaesyce } \\
\text { hypericifolia (L.) } \\
\text { Millspaugh }\end{array}$ & Lecherita, Lechera & $\begin{array}{l}\text { Whole plant, } \\
\text { fresh }\end{array}$ & Oral & Promoting lactation in women after birth & JULS67, GER41 \\
\hline $\begin{array}{l}\text { Manhiot esculenta } \\
\text { Crantz }\end{array}$ & Yuca & Tuber, fresh & Oral & Vaginal infection, Vaginal discharge & GER192 \\
\hline \multicolumn{6}{|l|}{ FABACEAE } \\
\hline $\begin{array}{l}\text { Caesalpinia spinosa } \\
\text { (Molina) Kuntze }\end{array}$ & Tara, Talla, Chanchalagua & $\begin{array}{l}\text { Seeds pods, } \\
\text { fresh or dried }\end{array}$ & Topical & $\begin{array}{l}\text { Fungus, Inflammation of ovaries, } \\
\text { Inflammation of uterus, Inflammation of } \\
\text { the vagina }\end{array}$ & $\begin{array}{l}\text { ISA55, EHCHL27, } \\
\text { VFCHL21, JULS255, } \\
\text { GER143 }\end{array}$ \\
\hline $\begin{array}{l}\text { Desmodium } \\
\text { molliculum (H.B.K.) DC. }\end{array}$ & $\begin{array}{l}\text { Pie de Perro, Pata-Perro, Pata } \\
\text { de Perro, Chancas de } \\
\text { Comida, Muña, Manayupa }\end{array}$ & $\begin{array}{l}\text { Whole plant, } \\
\text { fresh or dried }\end{array}$ & Topical & $\begin{array}{l}\text { Inflammation of the ovaries, Inflammation } \\
\text { of the womb }\end{array}$ & $\begin{array}{l}\text { JULS41, RBU/PL268, } \\
\text { GER135, JULS44, } \\
\text { EHCHL109, RBU/PL256 }\end{array}$ \\
\hline $\begin{array}{l}\text { Indigofera suffruticosa } \\
\text { Miller }\end{array}$ & Añil & Stems, fresh & Oral & $\begin{array}{l}\text { Cleaning of the woman, Expelling } \\
\text { placenta from woman after giving birth }\end{array}$ & GER198 \\
\hline Inga edulis C. Martius & Huaba, Pacae, Guava, Pacai & Flowers, fresh & Topical & Hair growth & JULS168, JULS304, GER17 \\
\hline Inga feuillei DC. & Huaba, Pacae, Guava, Pacai & Flowers, fresh & Topical & Hair growth & JULS168, JULS304, GER17 \\
\hline $\begin{array}{l}\text { Mimosa nothacacia } \\
\text { Barneby }\end{array}$ & Uña de Gato de la Costa & Bark, dried & Topical & Anus cyst, Vaginal pimples, Anal pimples & JULS265, GER199 \\
\hline $\begin{array}{l}\text { Prosopis pallida }(\mathrm{H} . \& \\
\text { B. ex Willd.) H.B.K. }\end{array}$ & Algarrobo & Seeds, dried & Oral & Sexual potency & JULS97, GER8 \\
\hline \multicolumn{6}{|l|}{ GENTIANACEAE } \\
\hline $\begin{array}{l}\text { Gentianella } \\
\text { bruneotricha (Gilg.) J.S. } \\
\text { Pringle. }\end{array}$ & Anga Macha & $\begin{array}{l}\text { Whole plant, } \\
\text { fresh }\end{array}$ & Oral & Infection of the uterus, After giving birth & JULS282 \\
\hline \multicolumn{6}{|l|}{ GERANIACEAE } \\
\hline $\begin{array}{l}\text { Pelargonium } \\
\text { odoratisimum (L.) } \\
\text { L'Herit. }\end{array}$ & $\begin{array}{l}\text { Malva de Oro, Malva de } \\
\text { Olor, Malva Olorosa }\end{array}$ & $\begin{array}{l}\text { Whole plant, } \\
\text { fresh or dried }\end{array}$ & Oral & $\begin{array}{l}\text { Inflammation of the ovaries, Inflammation } \\
\text { of the womb }\end{array}$ & $\begin{array}{l}\text { TRUVan/Erica14, TRUBH6, } \\
\text { EHCHL89, JULS188 }\end{array}$ \\
\hline $\begin{array}{l}\text { Pelargonium roseum } \\
\text { Willd. }\end{array}$ & Geranio & $\begin{array}{l}\text { Flowers and } \\
\text { Leaves, fresh }\end{array}$ & Oral & $\begin{array}{l}\text { Hemorrhages, Uterus pain, Inflammation } \\
\text { of the uterus }\end{array}$ & JULS84 \\
\hline \multicolumn{6}{|l|}{ ILLICIACEAE } \\
\hline Illicium verum Hook. f. & Anis Estrella & Seeds, dried & Oral & $\begin{array}{l}\text { Expel residues of feces in stomach of } \\
\text { newborn babies }\end{array}$ & JULS102 \\
\hline \multicolumn{6}{|l|}{ ISOETACEAE } \\
\hline Isoetes andina R. \& P. & Piri Piri & Stems, fresh & Oral & Male impotence & ISA100 \\
\hline \multicolumn{6}{|l|}{ KRAMERIACEAE } \\
\hline $\begin{array}{l}\text { Krameria lappacea } \\
\text { (Dombey) Berdet \& B. } \\
\text { Simpson }\end{array}$ & Ratania, Raima & $\begin{array}{l}\text { Leaves and } \\
\text { Root, fresh }\end{array}$ & Oral & Inflammation of the ovaries & JULS53 \\
\hline \multicolumn{6}{|l|}{ LAMIACEAE } \\
\hline $\begin{array}{l}\text { Lepechinia meyenii } \\
\text { (Walpers) Epling }\end{array}$ & Salvia, Salvia Real & $\begin{array}{l}\text { Whole plant, } \\
\text { fresh or dried }\end{array}$ & $\begin{array}{l}\text { 1. Oral } \\
2 . \\
\text { Topical }\end{array}$ & $\begin{array}{l}\text { 1. Menstruation } \\
\text { 2. Hair loss }\end{array}$ & $\begin{array}{l}\text { RBU/PL303, VFCHL17, } \\
\text { ISA91 }\end{array}$ \\
\hline
\end{tabular}


Table 3 Species encountered and used in Northern Peru for reproductive problems (Continued)

\begin{tabular}{|c|c|c|c|c|c|}
\hline Mentha spicata $\mathrm{L}$. & $\begin{array}{l}\text { Hierba Buena, Hierba Buena } \\
\text { Silvestre, Menta }\end{array}$ & $\begin{array}{l}\text { Whole plant, } \\
\text { fresh }\end{array}$ & Oral & Aphrodisiac & $\begin{array}{l}\text { RBU/PL308, EHCHL74, } \\
\text { RBU/PL267, JULS72, } \\
\text { VFCHL3, JULS20, GER15, } \\
\text { GER134, JULS20 }\end{array}$ \\
\hline Ocimum basilicum L. & $\begin{array}{l}\text { Albaca Mistura, Albaca } \\
\text { Negra, Albaca, Albaca } \\
\text { Morada, Albahaca (costa) }\end{array}$ & $\begin{array}{l}\text { Whole plant, } \\
\text { fresh }\end{array}$ & Oral & $\begin{array}{l}\text { 1. To promote dialation of the uterus, } \\
\text { Hasten delivery, Preventing infections } \\
\text { related to birth, Refreshing womb, } \\
\text { Reducing inflammation after birth } \\
\text { 2. After birth }\end{array}$ & $\begin{array}{l}\text { JULS54, EHCHL48, } \\
\text { VFCHL13, RBU/PL284, } \\
\text { TRUVan/Erica8, GER191 }\end{array}$ \\
\hline Origanum majorana L. & Mejorana & $\begin{array}{l}\text { Leaves and } \\
\text { Stems, fresh }\end{array}$ & Oral & Menstration & $\begin{array}{l}\text { EHCHL88, JULS19, RBU/ } \\
\text { PL317, GER165 }\end{array}$ \\
\hline Origanum vulgare L. & Oregano & $\begin{array}{l}\text { Leaves and } \\
\text { Stems, fresh } \\
\text { or dried }\end{array}$ & Oral & $\begin{array}{l}\text { Menstrual cramps, Menstration, Lower } \\
\text { stomach cramps related to PMS }\end{array}$ & JULS205, GER114 \\
\hline $\begin{array}{l}\text { Rosmarinus officinalis } \\
\text { L. }\end{array}$ & Romero, Romero Castilla & $\begin{array}{l}\text { Leaves, fresh } \\
\text { or dried }\end{array}$ & Topical & Hair loss & $\begin{array}{l}\text { RBU/PL329, ISA78, } \\
\text { TRUBH11, EHCHL3, } \\
\text { JULS27, VFCHL2, ISA105 }\end{array}$ \\
\hline Salvia discolor H.B.K. & $\begin{array}{l}\text { Palmeras (Chica), Llatama, } \\
\text { Yatama }\end{array}$ & Stems, fresh & $\begin{array}{l}\text { 1. } \\
\text { Topcial } \\
\text { 2. Oral }\end{array}$ & $\begin{array}{l}\text { 1. Preventing infections related to birth, } \\
\text { Fright/Susto in children } \\
\text { 2. Preventing infections related to birth }\end{array}$ & ISA93, ISA151(93a), ISA25 \\
\hline Salvia officinalis L. & Salvia & $\begin{array}{l}\text { Whole plant, } \\
\text { fresh or dried }\end{array}$ & Oral & Control and regulate menstrual cycle & JULS241 \\
\hline $\begin{array}{l}\text { Satureja pulchella (H.B. } \\
\text { K.) Briquet }\end{array}$ & Panizara, Panisara & $\begin{array}{l}\text { Leaves, fresh } \\
\text { or dried }\end{array}$ & Oral & Menstrual delay & GER148, JULS43 \\
\hline \multicolumn{6}{|l|}{ LAURACEAE } \\
\hline Persea americana Mill. & Palta & Seeds, fresh & Oral & $\begin{array}{l}\text { Contraceptive, Sterilization for women } \\
\text { only }\end{array}$ & JULS211, GER18 \\
\hline \multicolumn{6}{|l|}{ LINACEAE } \\
\hline Linum sativum $\mathrm{L}$. & Linaza & Seeds, dried & Oral & Inflammation of the prostate & EHCHL1599 \\
\hline Linum usitatissimum L. & Linaza & Seeds, dried & Oral & Inflammation of the prostate & JULS185, GER139 \\
\hline \multicolumn{6}{|l|}{ LOGANIACEAE } \\
\hline Buddleja utilis Kraenzl. & Flor Blanca & $\begin{array}{l}\text { Flowers, fresh } \\
\text { or dried }\end{array}$ & Oral & $\begin{array}{l}\text { Menstruation, Inflammation of the womb, } \\
\text { Ovarian cysts, Inflammation of uterus }\end{array}$ & $\begin{array}{l}\text { RBU/PL333, EHCHL38, } \\
\text { ISA60, JULS155, GER136 }\end{array}$ \\
\hline \multicolumn{6}{|l|}{ LORANTHACEAE } \\
\hline $\begin{array}{l}\text { Tristerix } \\
\text { longibracteatus (Des.) } \\
\text { Barlow \& Wiens }\end{array}$ & Suelda con Suelda & $\begin{array}{l}\text { Whole plant, } \\
\text { dried }\end{array}$ & Oral & Vaginal discharge (white or yellow) & JULS296, GER74 \\
\hline \multicolumn{6}{|l|}{ LYTHRACEAE } \\
\hline $\begin{array}{l}\text { Cuphea strigulosa H.B. } \\
\text { K. }\end{array}$ & $\begin{array}{l}\text { Lancetilla, Gacetilla, } \\
\text { Sanguinaria, Gansetilla, } \\
\text { Hierba del Toro }\end{array}$ & $\begin{array}{l}\text { Leaves and } \\
\text { Stems, fresh }\end{array}$ & Oral & Discharges & $\begin{array}{l}\text { GER104, EHCHL35, } \\
\text { VFCHL34, JULS33, ISA51, } \\
\text { RBU/PL259, EHCHL43, } \\
\text { JULS59, ISA53, GER147 }\end{array}$ \\
\hline \multicolumn{6}{|l|}{ MALVACEAE } \\
\hline Malva sylvestris L. & Malva (Chica), Malva Blanca & $\begin{array}{l}\text { Leaves and } \\
\text { Stems, fresh } \\
\text { or dried }\end{array}$ & Topical & Vaginal cleansing & VFCHL49, EHCHL29 \\
\hline \multicolumn{6}{|l|}{ MENISPERMACEAE } \\
\hline $\begin{array}{l}\text { Abuta grandiflora } \\
\text { (Mart.) Sand. }\end{array}$ & Abuta (male and female) & $\begin{array}{l}\text { Root and } \\
\text { Stems, fresh } \\
\text { or dried }\end{array}$ & Oral & Contraceptive & JULS88, RBU/PL312 \\
\hline \multicolumn{6}{|l|}{ MORACEAE } \\
\hline $\begin{array}{l}\text { Brosmium rubescens } \\
\text { Taubert }\end{array}$ & $\begin{array}{l}\text { Palo Sangre, Palo de la } \\
\text { Sangre, Ablita }\end{array}$ & $\begin{array}{l}\text { Wood and } \\
\text { Bark, fresh or } \\
\text { dried }\end{array}$ & Oral & $\begin{array}{l}\text { 1. Fertility, Sexual potency } \\
\text { 2. Haemorrhages (prevention and healing }\end{array}$ & $\begin{array}{l}\text { JULS209, ISA49, } \\
\text { EHCHL64, RBU/PL311, } \\
\text { GER86, EHCHL62 }\end{array}$ \\
\hline \multicolumn{6}{|l|}{ MYRISTICACEAE } \\
\hline Myristica fragrans L. & Nuez Moscada, Ajonjoli & Seeds, dried & Oral & Fertility, Sexual potency & $\begin{array}{l}\text { RBU/PL385, EHCHL155, } \\
\text { JULS292, GER197 }\end{array}$ \\
\hline \multicolumn{6}{|l|}{ NYCTAGINACEAE } \\
\hline Mirabilis jalapa L. & Buenas Tardes & Root, fresh & Oral & Prostate, Pre-prostate cancer & JULS116, GER185 \\
\hline
\end{tabular}


Table 3 Species encountered and used in Northern Peru for reproductive problems (Continued)

\begin{tabular}{|c|c|c|c|c|c|}
\hline \multicolumn{6}{|l|}{ OLACACEAE } \\
\hline $\begin{array}{l}\text { Heisteria acuminata } \\
(\mathrm{H} . \& \text { \&.) Engler }\end{array}$ & Chuchuasi, Chuchuhuasi & $\begin{array}{l}\text { Bark, fresh or } \\
\text { dried }\end{array}$ & Oral & Sexual potency & $\begin{array}{l}\text { RBU/PL287, JULS138, } \\
\text { GER164 }\end{array}$ \\
\hline Ximenia americana $\mathrm{L}$. & Limoncillo & $\begin{array}{l}\text { Whole plant, } \\
\text { fresh or dried }\end{array}$ & Oral & Menstrual regulation & JULS184 \\
\hline \multicolumn{6}{|l|}{ ORCHIDACEAE } \\
\hline $\begin{array}{l}\text { Aa paleacea (H.B.K.) } \\
\text { Rchb. f. }\end{array}$ & $\begin{array}{l}\text { Hierba de la Soledad, Hierba } \\
\text { Sola }\end{array}$ & Leaves, fresh & Oral & Contraceptive, Sterilization of women & ISA141, EHCHL75 \\
\hline \multicolumn{6}{|l|}{ OXALIDACEAE } \\
\hline Oxalis tuberosa Molina & Oca Rosada & Tuber, fresh & Oral & Sexual potency & JULS203 \\
\hline \multicolumn{6}{|c|}{ PASSIFLORACEAE } \\
\hline $\begin{array}{l}\text { Passiflora } \\
\text { quardrangularis L. }\end{array}$ & Hojas de Tumbo & Leaves, fresh & Oral & Menstrual pain & EHCHL135 \\
\hline Passiflora sp. & Chulgan & $\begin{array}{l}\text { Leaves and } \\
\text { Stems, dried }\end{array}$ & Oral & $\begin{array}{l}\text { Promoting vaginal dilation during } \\
\text { childbirth. }\end{array}$ & JULS279 \\
\hline \multicolumn{6}{|l|}{ PLANTAGINACEAE } \\
\hline Plantago major L. & Llantén & Leaves, fresh & Topical & Vaginal cleansing & $\begin{array}{l}\text { VFCHL50, EHCHL11, } \\
\text { TRUVan/Erica13 }\end{array}$ \\
\hline $\begin{array}{l}\text { Plantago sericea R. \& } \\
\text { P. var. lanuginosa } \\
\text { Grieseb. }\end{array}$ & Pajilla Blanca & $\begin{array}{l}\text { Whole plant, } \\
\text { fresh or dried }\end{array}$ & Oral & Vaginal discharge & JULS207 \\
\hline $\begin{array}{l}\text { Plantago sericea R. \& } \\
\text { P. subsp. sericans } \\
\text { (Pilger) Rahn }\end{array}$ & Paja Blanca & $\begin{array}{l}\text { Stems, fresh } \\
\text { or dried }\end{array}$ & Oral & $\begin{array}{l}\text { Ovarian pain, Inflammation of the ovaries, } \\
\text { Inflammation of the womb }\end{array}$ & RBU/PL335, EHCHL96 \\
\hline \multicolumn{6}{|l|}{ POACEAE } \\
\hline $\begin{array}{l}\text { Cynodon dactylon (L.) } \\
\text { Persoon }\end{array}$ & Grama Dulce & Stems, dried & Oral & $\begin{array}{l}\text { Cysts of the ovary, Cysts of the uterus, } \\
\text { Uterus, Fibroids, Uterus prolapse }\end{array}$ & $\begin{array}{l}\text { ISA61, JULS73, ISA106, } \\
\text { GER151 }\end{array}$ \\
\hline $\begin{array}{l}\text { Saccharum officinarum } \\
\mathrm{L} \text {. }\end{array}$ & $\begin{array}{l}\text { Azucar de Caña, Caña de } \\
\text { Azucar, Caña Dulce }\end{array}$ & $\begin{array}{l}\text { 1. Fresh sugar } \\
\text { 2. Stems, } \\
\text { fresh }\end{array}$ & $\begin{array}{l}\text { 1. } \\
\text { Topical } \\
\text { 2. Oral }\end{array}$ & $\begin{array}{l}\text { 1. Aphrodisiac } \\
\text { 2. Inflammation of the prostate }\end{array}$ & $\begin{array}{l}\text { VFCHL4, JULS123, } \\
\text { GER208 }\end{array}$ \\
\hline Triticum sativum $\mathrm{L}$. & Trigo & Seeds, dried & Topical & Vaginal infection, Vaginal discharge & GER182 \\
\hline \multicolumn{6}{|l|}{ POLYGONACEAE } \\
\hline Rumex crispus L. & $\begin{array}{l}\text { Acelga, Lengua de Vaca, } \\
\text { Hojas de Mala Hierba }\end{array}$ & $\begin{array}{l}\text { Whole plant, } \\
\text { fresh }\end{array}$ & $\begin{array}{l}\text { 1. Oral } \\
2 . \\
\text { Topical }\end{array}$ & $\begin{array}{l}\text { 1. Infection of the uterus } \\
\text { 2. Inflamation (internal woman parts), } \\
\text { Vaginal inflammation }\end{array}$ & JULS70, EHCHL173 \\
\hline \multicolumn{6}{|l|}{ POLYPODIACEAE } \\
\hline $\begin{array}{l}\text { Polypodium } \\
\text { crassifolium L. }\end{array}$ & Lengua de Ciervo, Calaguala & Stems, fresh & Oral & Prostate & $\begin{array}{l}\text { EHCHL71, TRUBH38, RBU/ } \\
\text { PL331, RBU/PL332, } \\
\text { JULS52, JULS303 }\end{array}$ \\
\hline \multicolumn{6}{|l|}{ PORTULACACEAE } \\
\hline Portulaca villosa H.B.K. & Verdolaga & $\begin{array}{l}\text { Root and } \\
\text { Stems, fresh }\end{array}$ & Topical & Hair loss & GER171 \\
\hline \multicolumn{6}{|l|}{ PROTEACEAE } \\
\hline $\begin{array}{l}\text { Oreocallis grandiflora } \\
\text { (Lam.) R.Br. }\end{array}$ & $\begin{array}{l}\text { Rumilanche, Bunbun, } \\
\text { Huaminga }\end{array}$ & $\begin{array}{l}\text { Leaves and } \\
\text { Stems, fresh } \\
\text { or dried }\end{array}$ & Oral & $\begin{array}{l}\text { Inflammation of the ovaries, Inflammation } \\
\text { of uterus }\end{array}$ & $\begin{array}{l}\text { EHCHL127, JULS31, } \\
\text { ISA28, ISA70 }\end{array}$ \\
\hline \multicolumn{6}{|l|}{ RANUNCULACEAE } \\
\hline $\begin{array}{l}\text { Laccopetalum } \\
\text { giganteum (Wedd.) } \\
\text { Ulbrich }\end{array}$ & $\begin{array}{l}\text { Huamanripa, Pacra, Flor de } \\
\text { Guarmarya }\end{array}$ & $\begin{array}{l}\text { Leaves, fresh } \\
\text { or dried }\end{array}$ & Oral & Fertilization (Heat Ovaries) & $\begin{array}{l}\text { VFCHL53, RBU/PL321, } \\
\text { EHCHL42, JULS284, } \\
\text { GER162 }\end{array}$ \\
\hline \multicolumn{6}{|l|}{ ROSACEAE } \\
\hline $\begin{array}{l}\text { Sanguisorba minor } \\
\text { Scop. }\end{array}$ & Pimpinela, Flor de Overa & $\begin{array}{l}\text { Whole plant, } \\
\text { fresh }\end{array}$ & Oral & Menstrual regulation & $\begin{array}{l}\text { EHCHL117, TRUBH35, } \\
\text { RBU/PL262, ISA57, } \\
\text { JULS25, ISA147(103a), } \\
\text { VFCHL20, GER170 }\end{array}$ \\
\hline \multicolumn{6}{|l|}{ RUBIACEAE } \\
\hline Cinchona officinalis $\mathrm{L}$. & Cascarilla, Quinuagiro & Bark, dried & Oral & Fertility, Sexual potency & $\begin{array}{l}\text { RBU/PL314, JULS127, } \\
\text { ISA19, GER167 }\end{array}$ \\
\hline
\end{tabular}


Table 3 Species encountered and used in Northern Peru for reproductive problems (Continued)

\begin{tabular}{|c|c|c|c|c|c|}
\hline \multicolumn{6}{|l|}{ RUTACEAE } \\
\hline Ruta graveolens $\mathrm{L}$. & $\begin{array}{l}\text { Ruda, Ruda (Macho y } \\
\text { Hembra), Hierba del Quinde }\end{array}$ & $\begin{array}{l}\text { Whole plant, } \\
\text { fresh }\end{array}$ & $\begin{array}{l}\text { 1. Oral } \\
\text { 2. } \\
\text { Topical }\end{array}$ & $\begin{array}{l}\text { 1. Abortion } \\
\text { 2. Aphrodisiac. }\end{array}$ & $\begin{array}{l}\text { ISA152, JULS1, TRUVan/ } \\
\text { Erica20, EHCHL128, } \\
\text { VFCHL16, ISA145(108a), } \\
\text { GER24 }\end{array}$ \\
\hline $\begin{array}{l}\text { Pouteria lucuma (R. \& } \\
\text { P.) Kuntze. }\end{array}$ & Lucuma & Fruit, fresh & Oral & $\begin{array}{l}\text { Promoting lactation on women after } \\
\text { giving birth }\end{array}$ & JULS186 \\
\hline \multicolumn{6}{|l|}{ SOLANACEAE } \\
\hline $\begin{array}{l}\text { Cestrum auriculatum } \\
\text { L'Herit }\end{array}$ & Hierba Santa, Agrasejo & $\begin{array}{l}\text { Leaves, fresh } \\
\text { or dried }\end{array}$ & Topical & $\begin{array}{l}\text { Preventing spasms after giving birth, } \\
\text { Warming women }\end{array}$ & $\begin{array}{l}\text { JULS166, RBU/PL281, } \\
\text { EHCHL172, ISA122, } \\
\text { GER174, EHCHL102 }\end{array}$ \\
\hline $\begin{array}{l}\text { Cestrum strigilatum } R \text {. } \\
\& P .\end{array}$ & Santa María & $\begin{array}{l}\text { Flowers, } \\
\text { leaves and } \\
\text { Stems, fresh } \\
\text { or dried }\end{array}$ & Oral & Control and regulate menstrual cycle & JULS245 \\
\hline $\begin{array}{l}\text { Cestrum undulatum } \mathrm{R} \text {. } \\
\& P \text {. }\end{array}$ & Santa María & $\begin{array}{l}\text { Flowers, } \\
\text { leaves and } \\
\text { Stems, fresh } \\
\text { or dried }\end{array}$ & Oral & Control and regulate menstrual cycle & JULS246 \\
\hline Solanum tuberosum L. & Chuno de Papa & Tuber, dried & Oral & After childbirth complications & JULS140, JULS141 \\
\hline \multicolumn{6}{|l|}{ THELYPTERIDACEAE } \\
\hline $\begin{array}{l}\text { Thelypteris cf. scalaris } \\
\text { (Christ.) Alton }\end{array}$ & Helecho Macho & $\begin{array}{l}\text { Whole plant, } \\
\text { fresh or dried }\end{array}$ & Oral & Contraceptive & JULS291 \\
\hline \multicolumn{6}{|l|}{ THYMELEACEAE } \\
\hline $\begin{array}{l}\text { Daphnopsis } \\
\text { weberbaueri Domke }\end{array}$ & Los Cholitos, Cholitos & Seeds, dried & Oral & Infertility in women & $\begin{array}{l}\text { EHCHL153, JULS137, } \\
\text { GER216 }\end{array}$ \\
\hline \multicolumn{6}{|l|}{ TYPHACEAE } \\
\hline Typha angustifolia L. & Chante & Stems, dried & Oral & Prostate & ISA45 \\
\hline \multicolumn{6}{|l|}{ URTICACEAE } \\
\hline $\begin{array}{l}\text { Pilea microphylla (L.) } \\
\text { Lieberman }\end{array}$ & Contra Hierba & $\begin{array}{l}\text { Whole plant, } \\
\text { fresh }\end{array}$ & Oral & Prostate, Cysts & RBU/PL282, EHCHL33 \\
\hline \multicolumn{6}{|l|}{ VALERIANACEAE } \\
\hline $\begin{array}{l}\text { Phyllactis rigida (R. \& } \\
\text { P.) Persoon }\end{array}$ & $\begin{array}{l}\text { Hornamo Estrella, Siete } \\
\text { Sabios, Valeriana Estrella, } \\
\text { Valeriana, Hierba de la } \\
\text { Estrella }\end{array}$ & Stems, fresh & Oral & Menopause & $\begin{array}{l}\text { EHCHL163, TRUBH30, } \\
\text { JULS57, EHCHL44, } \\
\text { JULS46, ISA137, RBU/ } \\
\text { PL365, RBU/PL355, } \\
\text { GER187 }\end{array}$ \\
\hline \multicolumn{6}{|l|}{ VERBENACEAE } \\
\hline $\begin{array}{l}\text { Lantana scabiosaefolia } \\
\text { H.B.K. }\end{array}$ & Mastrando, Mastrante & $\begin{array}{l}\text { Leaves and } \\
\text { Stems, fresh } \\
\text { or dried }\end{array}$ & Oral & $\begin{array}{l}\text { Cold of the ovaries, Menstruation, } \\
\text { Women after childbirth to avoid colds }\end{array}$ & VFCHL51, GER6 \\
\hline
\end{tabular}

afflictions, and some have been investigated for their efficacy with positive results. An often-limiting factor to these investigations is lack of comprehensive ethnobotanical data to help choose plant candidates for potency/ efficacy tests. Since the plant parts utilized in preparation of the remedies are reported in this survey, it serves as an indication of species that may need further ecological assessment on their regeneration status.

The results of this study show that both indigenous and introduced species are used for the treatment of reproductive system problems. The information gained on frequently used traditional remedies might give some leads for future targets for further analysis in order to develop new drugs. However, more detailed scientific studies are desperately needed to evaluate the efficacy and safety of the remedies employed traditionally.

\section{Declaration of competing interests}

The authors declare that they have no competing interests.

\section{Authors' contributions}

RB collected/identified plant material analysis of the data as well as writing the manuscript. AG conducted fieldwork, data analysis and manuscript composition. Both authors have read and approved the final manuscript.

\section{Acknowledgements}

The presented study was financed through MIRT/MHIRT (Minority Health Disparity International Research and Training) a grant from the National Institutes of Health (Fund: 54112B MHIRT Program, Grant: G0000613).

Fieldwork for this project was supported through the assistance of a large 
number of MIRT/MHIRT students and volunteers. Thanks to all of them. None of the work would have been possible without the invaluable collaboration of Douglas Sharon and our Peruvian colleagues, especially curanderas Julia Calderón, Isabel Chinguel, and Olinda Pintado, curanderos Germán Santisteban and Leoncio Carrión, and herbalists Manuel Bejarano, Elmer Cruz, and Iván Cruz. Thanks also go to Eric Rodriguez (Herbarium Truxillense, HUT) and Abundio Sagastegui, Segundo Leiva, and Mario Zapata (Herbario Antenor Orrego, HAO) for the use of their facilities and their assistance in plant identification.

Received: 1 June 2010 Accepted: 1 November 2010

Published: 1 November 2010

\section{References}

1. World Health Organization: Sexually transmitted infections fact sheet. Geneva, World Health Organization; 2007.

2. World Health Organization: Traditional, Complementary and Alternative Medicines and Therapies. Washington DC, WHO Regional Office for the Americas/Pan American Health Organization (Working group OPS/OMS); 1999.

3. World Health Organization: Report, Technical Briefing on TM. Forty-ninth Regional Committee Meeting, Manila, Philippines, 18 September 1998 Manila, WHO Regional Office for the Western Pacific; 1998

4. United Nations Conference on Trade and Development: Systems and National Experiences for Protecting Traditional Knowledge, Innovations and Practices. Background Note by the UNCTAD Secretariat Geneva, United Nations Conference on Trade and Development; 2000, document reference TD/B/COM.1/EM.13/2.

5. World Health Organization: Consultation Meeting on TM and Modern Medicine, Harmonizing the Two Approaches. Geneva, World Health Organization; 1999, document reference (WP)TM/ICP/TM/001/RB/98- RS/99/ $\mathrm{GE} / 32(\mathrm{CHN})$.

6. World Health Organization: WHO TM Strategy 2002-2005. World Health Organization, Geneva; 2002.

7. Breevort P: The Booming U. S. Botanical Market: A New Overview. HerbalGram 1998, 44:33-46.

8. Camino L: Cerros, plantas y lagunas poderosas, la medicina al norte de Perú. Lima, Lluvia Editores; 1992.

9. Bussmann RW, Sharon D: Traditional plant use in Northern Peru, Tracking two thousand years of health culture. Journal of Ethnobiology and Ethnomedicine 2006, 2:47.

10. de Feo V: Medicinal and magical plants on northern Peruvian Andes. Fitoterapia 1992, 63:417-440.

11. Polia M: Las Lagunas de los Encantos - Medicina Tradicional Andina en el Peru septentrional. Lima, CePeSer; 1988.

12. Sharon D: Wizard of the Four Winds, A Shaman's Story. Free Press, New York; 1978.

13. Sharon D, Bussmann RW: Plantas Medicinales en la Obra del Obispo Don Baltasar Jaime Martínez Compañon (Siglo XVIII). In Desde el exterior, El Perú y sus estudios. Edited by: Millones L, Kato T. Tercer Congreso Internacional de Peruanistas, Nagoya, UNMSM, FEFCS, Lima; 2006:147-165.

14. EsSalud/Organización Panamericana de Salud: Estudio Costo-Efectividad: Programa Nacional de Medicina Complementaria. Seguro Social de EsSalud (Study of Cost-Effectiveness: National Program in Complementary Medicine. Social Security of EsSalud) Lima, EsSalud/Organización Panamericana de Salud (Pan American Health Organization); 2000.

15. Brako L, Zarucchi JL: Catalogue of the Flowering Plants and Gymnosperms of Peru. Missouri Botanical Garden, Saint Louis, MO; 1993.

16. Jørgensen PM, León-Yanez S: Catalogue of the vascular plants of Ecuador. Monographs in Systematic Botany from the Missouri Botanical Garden 1999, 75.

17. McBride JF: Flora of Peru. Fieldiana, Botany Field Museum of Natural History, Chicago;1936-1981.

18. Jørgensen PM, Ulloa Ulloa C: Seed plants of the High Andes of Ecuador a Checklist. AAU Reports 1994, 34:1-443.

19. Pestalozzi HU: Flora ilustrada altoandina. Herbario Nacional de Bolivia and Herbario Forestal Nacional Martín Cardenas, Cochabamba; 1998.

20. Ulloa Ulloa C, Jørgensen PM: Arboles y arbustos de los Andes del Ecuador. AAU Reports 1993, 30:1-263.

21. Sparre G, Harling B: Flora of Ecuador. Council for Nordic Publications in Botany;1978-2009.
22. Telefo PB, Moundipa PF, Tchouanguep FM: Oestrogenicity and effect on hepatic metabolism of the aqueous extract of the leaf mixture of Aloe buettneri, Dicliptera verticillata, Hibiscus macranthus and Justicia insularis. Fitoterapia 2002, 73(6):472-478.

23. Lans C: Ethnomedicines used in Trinidad and Tobago for reproductive problems. Journal of Ethnobiology and Ethnomedicine 2007, 3:13.

24. Adams JD Jr, Garcia C: Women's health among the Chumash. Evidence Based Complementary and Alternative Medicine 2006, 3(1):125-131.

25. Macía MJ, García E, Vidaurre PJ: An ethnobotanical survey of medicinal plants commercialized in the markets of La Paz and El Alto, Bolivia. Journal of Ethnopharmacology 2005, 97(2):337-350.

26. Niederhofer $\mathrm{H}$ : Is phytotherapy effective in menopausal dysphoric disorder? Menopause 2009, 16(3):616, author reply 616-617.

27. Kupfersztain C, Rotem C, Fagot R, Kaplan B: The immediate effect of natural plant extract, Angelica sinensis and Matricaria chamomilla (Climex) for the treatment of hot flushes during menopause. A preliminary report. Clinical and Experimental Obstetrics and Gynecology 2003, 30(4):203-206.

28. Ali-Shtayeh MS, Yaniv Z, Mahajna J: Ethnobotanical survey in the Palestinian area: a classification of the healing potential of medicinal plants. Journal of Ethnopharmacology 2000, 73(1-2):221-232.

29. Greenlee H, Atkinson C, Stanczyk FZ, Lampe JW: A pilot and feasibility study on the effects of naturopathic botanical and dietary interventions on sex steroid hormone metabolism in premenopausal women. Cancer Epidemiology Biomarkers and Prevention 2007, 16(8):1601-1609.

30. Zhi X, Honda K, Ozaki K, Misugi T, Sumi T, Ishiko O: Dandelion T-1 extract up-regulates reproductive hormone receptor expression in mice. International Journal of Molecular Medicine 2007, 20(3):287-292.

31. Mishra A, Dogra JV, Singh JN, Jha OP: Post-coital antifertility activity of Annona squamosa and Ipomoea fistulosa. Planta Medica 1979, 35(3):283-285.

32. Bourdy G, Walter A: Maternity and medicinal plants in Vanuatu. I. The cycle of reproduction. Journal of Ethnopharmacology 1992, 37(3):179-196.

33. Komesaroff PA, Black CV, Cable V, Sudhir K: Effects of wild yam extract on menopausal symptoms, lipids and sex hormones in healthy menopausal women. Climacteric 2001, 4(2):144-150.

34. Russell L, Hicks GS, Low AK, Shepherd JM, Brown CA: Phytoestrogens: a viable option? Am Journal of Medicinal Science 2002, 324(4):185188.

35. Wu WH, Liu LY, Chung CJ, Jou HJ, Wang TA: Estrogenic effect of yam ingestion in healthy postmenopausal women. Journal of the American College of Nutrition 2005, 24(4):235-243.

36. Parton K, Gardner D, Williamson NB: Isocupressic acid, an abortifacient component of Cupressus macrocarpa. Nwe Zealand Veterinary Journal 1996, 44(3):109-111.

37. Tsai YS, Tong YC, Cheng JT, Lee CH, Yang FS, Lee HY: Pumpkin seed oil and phytosterol- $\mathrm{F}$ can block testosterone/prazosin-induced prostate growth in rats. Urology International 2006, 77(3):269-274.

38. Lee YH, Hyun SH, Choung SY: Effect of herbal extract mixture on menopausal urinary incontinence in ovariectomized rats. Biofactors 2006, 26(3):171-178

39. Gossell-Williams M, Davis A, O'Connor N: Inhibition of testosteroneinduced hyperplasia of the prostate of sprague-dawley rats by pumpkin seed oil. Journal of Medicinal Food 2006, 9(2):284-286.

40. Nusier MK, Bataineh HN, Daradkah HM: Adverse effects of rosemary (Rosmarinus officinalis L.) on reproductive function in adult male rats. Experimental Biology and Medicine (Maywood) 2007, 232(6):809-813.

41. Chaudhury RR: The quest for a herbal contraceptive. Natlional Medical Journal of India 1993, 6(5):199-201.

42. Sharma N, Jacob D: Assessment of reversible contraceptive efficacy of methanol extract of Mentha arvensis L. leaves in male albino mice. Journal of Ethnopharmacology 2002, 80(1):9-13.

43. Sharma N, Jacob D: Antifertility investigation and toxicological screening of the petroleum ether extract of the leaves of Mentha arvensis L. in male albino mice. Journal of Ethnopharmacology 2001, 75(1):5-12.

44. Ciganda C, Laborde A: Herbal infusions used for induced abortion. Journal of Toxicology and Clinical Toxicology 2003, 41(3):235-239.

45. Batta SK, Santhakumari G: The antifertility effect of Ocimum sanctum and hibiscus Rosa sinensis. Indian Journal of Medical Research 1971, 59(5):777-781.

46. Kasinathan S, Ramakrishnan S, Basu SL: Antifertility effect of Ocimum sanctum L. Indian Journal of Experimental Biology 1972, 10(1):23-25. 
47. Farnsworth NR, Waller DP: Current status of plant products reported to inhibit sperm. Research Frontiers in Fertility Regulation 1982, 2(1):1-16.

48. Ahmed M, Ahamed RN, Aladakatti RH, Ghosesawar MG: Reversible antifertility effect of benzene extract of Ocimum sanctum leaves on sperm parameters and fructose content in rats. Journal of Basic Clinical Physiology and Pharmacology 2002, 13(1):51-59.

49. Allan P, Bilkei G: Oregano improves reproductive performance of sows. Theriogenology 2005, 63(3):716-721.

50. El-Ashmawy IM, Saleh A, Salama OM: Effects of marjoram volatile oil and grape seed extract on ethanol toxicity in male rats. Basic Clinical Pharmacology and Toxicology 2007, 101(5):320-327.

51. East J: The effect of certain plant preparations on the fertility of laboratory mammals. 4. Sanguisorba officinalis L. Journal of Endocrinoogyl 1955, 12(4):273-276.

52. Conway GA, Slocumb JC: Plants used as abortifacients and emmenagogues by Spanish New Mexicans. Journal of Ethnopharmacology 1979, 1(3):241-261

53. Gutiérrez-Pajares $\mathrm{J}$, Zúñiga $\mathrm{L}$, Pino J: Ruta graveolens aqueous extract retards mouse preimplantation embryo development. Reproductive Toxicology 2003, 17(6):667-672.

54. de Freitas TG, Augusto PM, Montanari T: Effect of Ruta graveolens L. on pregnant mice. Contraception 2005, 71(1):74-77.

55. Khouri NA, El-Akawi Z: Antiandrogenic activity of Ruta graveolens L in male Albino rats with emphasis on sexual and aggressive behavior. Neurology and Endocrinology Letters 2005, 26(6):823-829.

56. Dhawan K, Kumar S, Sharma A: Aphrodisiac activity of methanol extract of leaves of Passiflora incarnata Linn in mice. Phytotherapy Research 2003, 17(4):401-403.

57. Dhawan K, Kumar S, Sharma A: Beneficial effects of chrysin and benzoflavone on virility in 2-year-old male rats. Journal of Medicinal Food 2002, 5(1):434-8

58. Dhawan K, Sharma A: Restoration of chronic-Delta 9-THC-induced decline in sexuality in male rats by a novel benzoflavone moiety from Passiflora incarnata Linn. British Journal of Pharmacology 2003, 138(1):117-120.

59. Dhawan K: Drug/substance reversal effects of a novel tri-substituted benzoflavone moiety (BZF) isolated from Passiflora incarnata Linn.-a brief perspective. Addiction Biology 2003, 8(4):379-386.

60. Tabach R, Rodrigues E, Carlini EA: Preclinical toxicological assessment of a phytotherapeutic product-CPV (based on dry extracts of Crataegus oxyacantha L., Passiflora incarnata L., and Valeriana officinalis L.). Phytotherapy Research 2009, 23(1):33-40.

61. Tajuddin Ahmad S, Latif A, Qasmi IA, Amin KM: An experimental study of sexual function improving effect of Myristica fragrans Houtt. (nutmeg). BMC Complementary and Alternative Medicine 2005, 5:16.

62. Tajuddin Ahmad S, Latif A, Qasmi IA: Aphrodisiac activity of 50\% ethanolic extracts of Myristica fragrans Houtt. (nutmeg) and Syzygium aromaticum (L) Merr. \& Perry. (clove) in male mice: a comparative study. BMC Complementary and Alternative Medicine 2003, 3:6.

63. de Mello FB, Jacobus D, Carvalho K, Mello JR: Effects of Lantana camara (Verbenaceae) on general reproductive performance and teratology in rats. Toxicon 2005, 45(4):459-466.

64. de Mello FB, Jacobus D, de Carvalho KC, de Mello JR: Effects of Lantana camara (Verbenaceae) on rat fertility. Veterinary and Human Toxicology 2003, 45(1):20-23.

65. Verma OP, Kumar S, Chatterjee SN: Antifertility effects of common edible Portulaca oleracea on the reproductive organs of male albino mice. Indian Journal of Medical Research 1982, 75:301-310.

66. Shobeiri SF, Sharei S, Heidari A, Kianbakht S: Portulaca oleracea L. in the treatment of patients with abnormal uterine bleeding: a pilot clinical trial. Phytotherapy Research 2009, 23(10):1411-1414.

doi:10.1186/1746-4269-6-30

Cite this article as: Bussmann and Glenn: Medicinal plants used in

Northern Peru for reproductive problems and female health. Journal of Ethnobiology and Ethnomedicine 2010 6:30.

\section{Submit your next manuscript to BioMed Central and take full advantage of:}

- Convenient online submission

- Thorough peer review

- No space constraints or color figure charges

- Immediate publication on acceptance

- Inclusion in PubMed, CAS, Scopus and Google Scholar

- Research which is freely available for redistribution 